\title{
Quantum Graphology
}

\author{
T. Kottos \\ Max-Planck-Institute for Dynamics and Self-Organization \\ 37073 Göttingen, Germany
}

\begin{abstract}
We review quantum chaos on graphs. We construct a unitary operator which represents the quantum evolution on the graph and study its spectral and wave function statistics. This operator is the analogue of the classical evolution operator on the graph. It allows us to establish a connection between the corresponding periodic orbits and the statistical properties of eigenvalues and eigenfunctions. Specifically, for the energy-averaged spectral form factor we derived an exact combinatorial expression which illustrate the role of correlations between families of isometric orbits. We also show that enhanced wave function localization due to the presence of short unstable periodic orbits and strong scarring can rely on completely different mechanisms.
\end{abstract}

PACS numbers: 05.45.Mt, 03.65.Sq

\section{Introduction}

Quantum graphs have recently attracted a lot of interest [1-19]. A special volume containing a number of contributions can be found in [18]. The attention is due to the fact that quantum graphs can be viewed as typical and simple examples for the large class of systems in which classically chaotic dynamics implies universal spectral correlations in the semiclassical limit [20,21]. Up to now we have only a very limited understanding of the reasons for this universality. In a semiclassical approach to this problem the main stumbling block is the intricate interference between the contributions of (exponentially many) periodic orbits [22, 23]. Using quantum graphs as model systems it is possible to pinpoint and isolate this central problem. In graphs, an exact trace formula exists which is based on the periodic orbits of a mixing classical dynamical system [1,24]. Moreover the orbits can be specified by a finite symbolic code with Markovian grammar. Based on these simplifications it is possible to rewrite the spectral form factor or any other two-point correlation functions in terms of a combinatorial problem $[1,2,6,9]$. This combinatorial problem on graphs has been solved with promising results: It was shown that the form factor, ensemble averaged over graphs with a single non-trivial vertex and two attached bonds (2-Hydra) coincides exactly with the random-matrix 
result for the $2 \times 2$ circular unitary ensemble (CUE) [2]. A simple algorithm which can evaluate the resulting combinatorial sum for any graph was presented in [9]. In [3] the short-time expansion of the form factor for $v$-Hydra graphs (i.e. one central node with $v$ bonds attached) was computed in the limit $N \rightarrow \infty$. In [6] a periodic-orbit sum was used to prove Anderson localization in an infinite chain graph with randomized bond lengths. In [8] the form factor of binary graphs was shown to approach the random-matrix prediction when the number of vertices increases. In [11] the second order contribution $-2 \tau^{2}$ to the form factor was derived and was shown to be related to correlations within pairs of orbits differing in the orientation of one of the two loops resulting from a self-intersection of the orbit. Finally, in [16] a field theoretical method was used to evaluate exactly the form factor of large graphs. Very recently, the spectral properties of quantum graphs were studied experimentally by the Warsaw group [17] who constructed a microwave graph network.

The transport properties of open quantum graphs were also investigated quite thoroughly. In [7] compact graphs were connected with leads to infinity and was shown that they display all the features which characterize quantum chaotic scattering. In [14] the open quantum graphs were used to calculate shot-noise corrections while in [19] the same system was employed in order to understand current relaxation phenomena from open chaotic systems.

Quite recently the interest on quantum graphs moved towards understanding statistical properties of wave functions. In [15] the statistics of the nodal points was analyzed, while in [10, 13] quantum graphs were used in order to understand scaring of quantum eigenstates. A scar is a quantum eigenfunction with excess density near an unstable classical periodic orbit (PO). Such states are not expected within random-matrix theory (RMT), which predicts that wave functions must be evenly distributed over phase space, up to quantum fluctuations [25]. Experimental evidence and applications of scars come from systems as diverse as microwave resonators [26], quantum wells in a magnetic field [27], Faraday waves in confined geometries [28], open quantum dots [29], and semiconductor diode lasers [30].

This contribution, is structured in the following way. In the following Sect. 2, the main definitions and properties of quantum graphs are given. We concentrate on the unitary bond-scattering matrix $U$ which can be interpreted as a quantum evolution operator on the graph. Section 3 deals with the corresponding classical dynamical system. In Sect. 4, the statistical properties of the eigenphase spectrum of the bond-scattering matrix $U$ are analyzed and related to the periodic orbits of the classical dynamics. Scaring phenomenon is discussed and analyzed in Sect. 5. Finally, our conclusions and outlook are summarized in the last Sect. 6 .

\section{Quantum graphs: basic facts}

We start with a presentation and discussion of the Schr?odinger operator for graphs. Graphs consist of $V$ vertices connected by $B$ bonds. The valency $v_{i}$ 
of a vertex $i$ is the number of bonds meeting at that vertex. The graph is called $v$-regular if all the vertices have the same valency $v$. When the vertices $i$ and $j$ are connected, we denote the connecting bond by $b=(i, j)$. The same bond can also be referred to as $\vec{b} \equiv(\operatorname{Min}(i, j), \operatorname{Max}(i, j))$ or $\overleftarrow{b} \equiv(\operatorname{Max}(i, j), \operatorname{Min}(i, j))$ whenever we need to assign a direction to the bond. A bond with coinciding endpoints is called a loop. Finally, a graph is called bipartite if the vertices can be divided into two disjoint groups such that any vertices belonging to the same group are not connected.

Associated to every graph is its connectivity (adjacency) matrix $C_{i, j}$. It is a square matrix of size $V$ whose matrix elements $C_{i, j}$ are given in the following way:

$$
C_{i, j}=C_{j, i}= \begin{cases}1 & \text { if } i, j \text { are connected } \\ 0 & \text { otherwise }\end{cases}
$$

For graphs without loops the diagonal elements of $C$ are zero. The connectivity matrix of connected graphs cannot be written as a block diagonal matrix. The valency of a vertex is given in terms of the connectivity matrix, by $v_{i}=\sum_{j=1}^{V} C_{i, j}$ and the total number of indirected bonds is $B=\frac{1}{2} \sum_{i, j=1}^{V} C_{i, j}$.

For the quantum description we assign to each bond $b=(i, j)$ a coordinate $x_{i, j}$ which indicates the position along the bond. $x_{i, j}$ takes the value 0 at the vertex $i$ and the value $L_{i, j} \equiv L_{j, i}$ at the vertex $j$ while $x_{j, i}$ is zero at $j$ and $L_{i, j}$ at $i$. We have thus defined the length matrix $L_{i, j}$ with matrix elements different from zero, whenever $C_{i, j} \neq 0$ and $L_{i, j}=L_{j, i}$ for $b=1, \ldots, B$. The wave function $\Psi$ contains $B$ components $\Psi_{b_{1}}\left(x_{b_{1}}\right), \Psi_{b_{2}}\left(x_{b_{2}}\right), \ldots \Psi_{b_{B}}\left(x_{b_{B}}\right)$ where the set $\left\{b_{i}\right\}_{i=1}^{B}$ consists of $B$ different indirected bonds.

The Schrödinger operator (with $\hbar=2 m=1$ ) is defined on a graph in the following way: On each bond $b$, the component $\Psi_{b}$ of the total wave function $\Psi$ is a solution of the one-dimensional equation

$$
\left(-\mathrm{i} \frac{\mathrm{d}}{\mathrm{d} x}-A_{b}\right)^{2} \Psi_{b}(x)=k^{2} \Psi_{b}(x)
$$

We included a "magnetic vector potential" $A_{b}$ (with $\operatorname{Re}\left(A_{b}\right) \neq 0$ and $A_{\vec{b}}=-A_{\leftarrow}$ ) which breaks the time reversal symmetry. In most applications we shall assume that all the $A_{b}$ 's are equal and the bond index will be dropped. On each of the bonds, the general solution of (1) is a superposition of two counter propagating waves

$$
\Psi_{b=(i, j)}=a_{i, j} \exp \left(\mathrm{i}\left(k+A_{i, j}\right) x_{i, j}\right)+a_{j, i} \exp \left(\mathrm{i}\left(k+A_{j, i}\right) x_{j, i}\right) .
$$

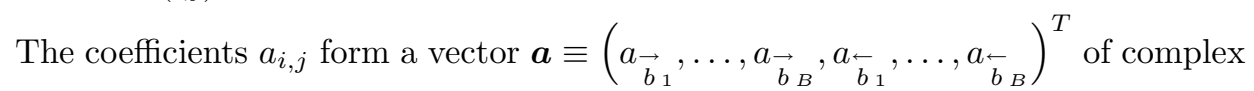
numbers which uniquely determines an element in a $2 B$-dimensional Hilbert space. This space corresponds to "free wave" solutions since we did not yet impose any conditions which the solutions of (1) have to satisfy at the vertices. 
The most general boundary conditions at the vertices are given in terms of unitary $v_{j} \times v_{j}$ vertex-scattering matrices $\sigma_{l, m}^{(l)}(k)$, where $l$ and $m$ go over all the vertices which are connected to $j$. At each vertex $j$, incoming and outgoing components of the wave function are related by

$$
a_{j, l}=\sum_{m=1}^{v_{j}} \sigma_{l, m}^{(j)}(k) \exp \left(\mathrm{i} k L_{j m}\right) a_{m, j},
$$

which implies current conservation. The particular form

$$
\sigma_{l, m}^{(j)}=\frac{2}{v_{j}}-\delta_{l, m}
$$

for the vertex-scattering matrices was shown in [1] to be compatible with continuity of the wave function and current conservation at the vertices. Equation (4) is referred to as Neumann boundary conditions. Bellow, we will concentrate on this type of graphs. Moreover we will always assume fully connected graphs, i.e. the valency is $v_{j}=v=V-1, \forall j=1, \ldots, V$.

Stationary states of the graph satisfy (3) at each vertex. These conditions can be combined into

$$
\boldsymbol{a}=U(k) \boldsymbol{a},
$$

such that the secular equation determining the eigenenergies and the corresponding eigenfunctions of the graph is of the form [1]

$$
\operatorname{det}[I-U(k, A)]=0 .
$$

Here, the unitary bond-scattering matrix

$$
U(k, A)=D(k ; A) T
$$

acting in the $2 B$-dimensional space of directed bonds has been introduced. The matrices $D$ and $T$ are given by

$$
\begin{aligned}
& D_{i j, i^{\prime} j^{\prime}}(k, A)=\delta_{i, i^{\prime}} \delta_{j, j^{\prime}} \exp \left(\mathrm{i} k L_{i j}+\mathrm{i} A_{i, j} L_{i j}\right) ; \\
& T_{j i, n m}=\delta_{n, i} C_{j, i} C_{i, m} \sigma_{j, m}^{(i)} .
\end{aligned}
$$

$T$ contains the topology of the graph and is equivalent to the complete set of vertex-scattering matrices, while $D$ contains the metric information about the bonds. Hereafter, the bond lengths $L_{m}(m=1, \ldots, B)$ will be chosen to be incommensurate in order to avoid non-generic degeneracies.

It is instructive to interpret the action of $U$ on an arbitrary graph state as its time evolution over an interval corresponding to the mean bond length of the graph such that

$$
\boldsymbol{a}(t)=U^{t} \boldsymbol{a}(0), \quad t=0,1,2, \ldots
$$

Clearly the solutions of (5) are stationary with respect to this time evolution. $n$ in (9) represents a discrete (topological) time counting the collisions of the particle with vertices of the graph. In this "picture" the diagonal matrix $D_{m n}(k)=$ $\delta_{m n} \exp \left(\mathrm{i} k l_{m}\right)$ describes the free propagation along the bonds of the network while 
$T$ assigns a scattering amplitude for transitions between connected directed bonds. As we will see in the next section it specifies a Markovian random walk on the graph which is the classical analogue of Eq. (9).

\section{Periodic orbits and classical dynamics on graphs}

In this section we discuss the classical dynamics corresponding to the quantum evolution (9) implied by $U$. To introduce this dynamics we employ a Liouvillian approach, where a classical evolution operator assigns transition probabilities in a phase space of $2 B$ directed bonds [1]. If $\rho_{b}(t)$ denotes the probability to occupy the (directed) bond $b$ at the (discrete) topological time $t$, we can write down a Markovian master equation of the form

$$
\rho_{b}(t+1)=\sum_{b^{\prime}} M_{b, b^{\prime}} \rho_{b^{\prime}}(t) .
$$

The classical (Frobenius-Perron) evolution operator $M$ has matrix elements

$$
M_{i j, n m}=\delta_{j, n} P_{i \rightarrow m}^{(j)}
$$

with $P_{j i \rightarrow i j^{\prime}}^{(i)}$ denoting the transition probability between the directed bonds $b=(j, i)$ and $b^{\prime}=\left(i, j^{\prime}\right)$. To make the connection with the quantum description, we adopt the quantum transition probabilities, expressed as the absolute squares of matrix elements of $M$

$$
P_{j \rightarrow j^{\prime}}^{(i)}=\left|\sigma_{j, j^{\prime}}^{(i)}(k)\right|^{2} \text {. }
$$

Let us note that $P_{j \rightarrow j^{\prime}}^{(i)}$ and $M$ do not involve any metric information on the graph.

The unitarity of the bond-scattering matrix $U$ guarantees $\sum_{b=1}^{2 B} M_{b, b^{\prime}}=1$ and $0 \leq M_{b, b^{\prime}} \leq 1$, so that the total probability that the particle is on any bond remains conserved during the evolution. The spectrum of $M$, denoted as $\left\{\mu_{b}\right\}$ with $b=1, \ldots, 2 B$, is restricted to the interior of the unit circle and $\mu_{1}=1$ is always an eigenvalue with the corresponding eigenvector $|1\rangle=\frac{1}{2 B}(1,1, \ldots, 1)^{T}$. In most cases, the eigenvalue 1 is the only eigenvalue on the unit circle. Then, the evolution is ergodic since any initial density will evolve to the eigenvector $|1\rangle$ which corresponds to a uniform distribution (equilibrium). The rate at which equilibrium is approached is determined by the gap to the next largest eigenvalue. If this gap exists, the dynamics is also mixing.

It was shown recently [16] that mixing dynamics alone does not suffice to guarantee universality of the spectral statistics of quantum graphs*. An additional condition proven recently by Gnutzmann and Altland [16] states that in the limit of $B \rightarrow \infty$, the spectral gap has to be constant or at least vanish slowly enough as $\Delta_{g} \equiv\left(1-\left|\mu_{2}\right|\right) \propto B^{-\alpha}$ with $0 \leq \alpha<0.5$ and $\mu_{2}$ being the second maximum eigenvalue of $M$. In Fig. 1 we report our numerical results for Neumann fully connected graphs. We see that this type of graph satisfies the condition requested by $[16]$.

\footnotetext{
${ }^{*}$ For an example of a mixing graph with non-universal spectral statistics, see [3].
} 

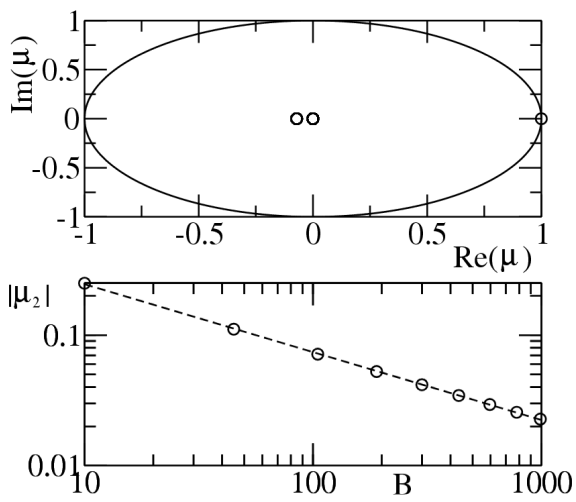

Fig. 1. Upper part: The spectrum of the classical evolution operator $M$ for the case of $V=15$ fully connected graph. Lower part: The scaling of the second maximum eigenvalue $\left|\mu_{2}\right|$ with respect to $B$. Solid line is the best linear fitting indicating that $\left|\mu_{2}\right| \propto B^{-0.5}$.

Graphs are one-dimensional and the motion on the bonds is simple and stable. Ergodic (mixing) dynamics is generated because at each vertex a (Markovian) choice of one out of $v$ directions is made. Thus, chaos on graphs originates from the multiple connectivity of the (otherwise linear) system [1].

Despite the probabilistic nature of the classical dynamics, the concept of a classical orbit can be introduced. A classical orbit on a graph is an itinerary of successively connected directed bonds $\left(i_{1}, i_{2}\right),\left(i_{2}, i_{3}\right), \ldots$ An orbit is periodic with period $t_{p}$ if for all $k,\left(i_{t_{p}+k}, i_{t_{p}+k+1}\right)=\left(i_{k}, i_{k+1}\right)$. For graphs without loops or multiple bonds, the sequence of vertices $i_{1}, i_{2}, \ldots$ with $i_{m} \in[1, V]$ and $C_{i_{m}, i_{m+1}}=1$ for all $m$ represents a unique code for the orbit. This is a finite coding which is governed by a Markovian grammar provided by the connectivity matrix. In this sense, the symbolic dynamics on the graph is Bernoulli. This analogy is strengthened by further evidence: The number of $t_{p}$-PO's on the graph is $\frac{1}{t_{p}} \operatorname{tr} C^{t_{p}}$, where $C$ is the connectivity matrix. Since its largest eigenvalue $\Gamma_{c}$ is bounded between the minimum and the maximum valency, i.e. $\min v_{i} \leq \Gamma_{c} \leq \max v_{i}$, periodic orbits proliferate exponentially with topological entropy $\approx \log T_{c}$.

From the previous discussion it is clear that all periodic orbits on a graph are unstable. The classical probability to remain at a specific $\mathrm{PO}$ of period $t_{p}$ is $M_{p}=\prod_{t=1}^{t_{p}}\left(M^{t}\right)_{j, j}$. As $M_{p}<1$, the probability to follow the PO decreases exponentially with time. Assuming regular graphs of valency $v_{j}=v$ we can evaluate the rate of instability as

$$
M_{p}=\prod_{s=1}^{r_{p}}\left(1-\frac{2}{v}\right)^{2} \prod_{f=1}^{t_{p}-r_{p}}\left(\frac{2}{v}\right)^{2} \equiv \exp \left(-\Lambda_{p} t_{p}\right),
$$

where $\Lambda_{p}$ plays the role of the Lyapunov exponent (LE) and $r_{p}$ is the number of vertices where back scattering occurs. For the graphs studied in this contribution, 


\begin{tabular}{llll}
\hline $\mathrm{t}_{\mathrm{p}}$ & $\mathrm{p}$ & \multicolumn{1}{c}{$\mathrm{M}_{\mathrm{p}}$} & $\Lambda_{\mathrm{p}}(\mathrm{v} \rightarrow \infty)$ \\
2 & $\bullet$ & $(2 / \mathrm{v}-1)^{4}$ & $4 / \mathrm{v}$ \\
3 & $\triangle$ & $(2 / \mathrm{v})^{6}$ & $2 \ln (\mathrm{v})$ \\
4 & ${ }_{(2 / \mathrm{v})^{4}}(2 / \mathrm{v}-1)^{4}$ & $\ln (\mathrm{v})$ \\
& $\square$ & $(2 / \mathrm{v})^{8}$ & $2 \ln (\mathrm{v})$
\end{tabular}

Fig. 2. The topology of the shortest PO's of a fully connected graph with valency $v$ is shown together with the classical probabilities to remain, and their corresponding Lyapunov exponent.

some PO's $p$ and LE $\Lambda_{p}$, are listed in Fig. 2. The shortest PO's have period 2 and bounce back and forth between two vertices. For large graphs $v \rightarrow \infty$ these are by far the least unstable ones, as their LE approaches 0 while all others become increasingly unstable $\Lambda_{p} \sim \ln v$.

\section{The spectral statistics of $U$}

We consider the matrix $U(k, A)$ defined in Eqs. $(7,8)$. The spectrum consists of $2 B$ points $\exp \left(\mathrm{i}_{l}(k)\right)$ confined to the unit circle (eigenphases). Unitary matrices of this type are frequently studied since they are the quantum analogues of classical, area preserving maps. Their spectral fluctuations depend on the nature of the underlying classical dynamics [31]. The quantum analogues of classically integrable maps display Poissonian statistics while in the opposite case of classically chaotic maps, the eigenphase statistics conform with the results of RMT for Dyson's circular ensembles. To describe the spectral fluctuations of $U$ we consider the form factor

$$
K(t, 2 B)=\frac{1}{2 B}\left\langle\left|\operatorname{tr} U^{t}\right|^{2}\right\rangle \quad(t>0) .
$$

The average $\langle\ldots\rangle$ will be specified below. RMT predicts that $K(t, 2 B)$ depends on the scaled time $\tau=t / 2 B$ only [31], and explicit expressions for the orthogonal and the unitary circular ensembles are known [25].

Using (7), (8) we expand the matrix products in $\operatorname{tr} U^{t}$ and obtain a sum of the form

$$
\operatorname{tr} U^{t}(k)=\sum_{p \in \mathcal{P}_{t}} \mathcal{A}_{p} \exp \left(\mathrm{i}\left(k L_{p}+A l_{p}\right)\right) .
$$

In this sum $p$ runs over all closed trajectories on the graph which are compatible with the connectivity matrix and which have the topological length $t$, i.e. they visit exactly $t$ vertices. For graphs, the concepts of closed trajectories and periodic orbits coincide, hence (15) can also be interpreted as a periodic-orbit sum. From (15) it is clear that $K(t / 2 B)=0$ as long as $t$ is smaller than the period of the shortest periodic orbit. The phase associated with an orbit is determined by its 
total (metric) length $L_{p}=\sum_{b \in p} L_{b}$ and by the "magnetic flux" through the orbit. The latter is given in terms of its total directed length $l_{p}$ if we assume for simplicity that the magnitude of the magnetic vector potential is constant $\left|A_{b}\right| \equiv A$. The amplitude of the contribution from a periodic orbit by the product of all the elements of vertex-scattering matrices encountered

$$
\mathcal{A}_{p}=\prod_{j=1}^{n_{p}} \sigma_{i_{j-1}, i_{j+1}}^{\left(i_{j}\right)} \equiv \prod_{[r, s, t]}\left(\sigma_{r, t}^{(s)}\right)^{n_{p}(r, s, t)}
$$

i.e. for fixed boundary conditions at the vertices it is completely specified by the frequencies $n_{p}(r, s, t)$ of all transitions $(r, s) \rightarrow(s, t)$. Inserting (15) into the definition of the form factor we obtain a double sum over periodic orbits

$$
K(t / 2 B)=\frac{1}{2 B}\left\langle\sum_{p, p^{\prime} \in \mathcal{P}_{n}} \mathcal{A}_{p} \mathcal{A}_{p l}^{*} \exp \left(\mathrm{i} k\left(L_{p}-L_{p^{\prime}}\right)+\mathrm{i} A\left(l_{p}-l_{p^{\prime}}\right)\right)\right\rangle .
$$

Now we have to specify our averaging procedure which has to respect the restrictions imposed by the underlying classical dynamics. To this end we will use the wave number $k$ for averaging, i.e. $\langle\ldots\rangle_{k}=\lim _{k \rightarrow \infty} k^{-1} \int_{0}^{k} \mathrm{~d} k^{\prime}(\ldots)$ (and, if present, also the magnetic vector potential $A$ ). Provided that the bond lengths of the graph are rationally independent and that a sufficiently large interval is used for averaging, we have

$$
\left\langle\operatorname { e x p } ( \mathrm { i } k ( L _ { p } - L _ { p ^ { \prime } } ) \rangle _ { k } = \delta _ { L _ { p } , L _ { p ^ { \prime } } } \quad \text { and } \quad \left\langle\exp \left(\mathrm{i} A\left(l_{p}-l_{p^{\prime}}\right)\right\rangle_{A}=\delta_{l_{p}, l_{p^{\prime}}},\right.\right.
$$

i.e. only terms with $L_{p}=L_{p^{\prime}}$ and $l_{p}=l_{p^{\prime}}$ survive.

Note that $L_{p}=L_{p^{\prime}}$ does not necessarily imply $p=p^{\prime}$ or that $p, p^{\prime}$ are related by some symmetry because there exist families $\mathcal{L}$ of distinct but isometric orbits which can be used to write the result of (17) in the form $[1,2,3,9]$

$$
K(t / 2 B)=\sum_{\mathcal{L} \in \mathcal{F}_{n}}\left|\sum_{p \in \mathcal{L}} \mathcal{A}_{p}\right|^{2}
$$

The outer sum is over the set $\mathcal{F}_{n}$ of families, while the inner one is a coherent sum over the orbits belonging to a given family (= metric length). An example of such family for the tetrahedron is shown in Fig. 3. Equation (19) is exact, and it represents a combinatorial problem since it does not depend any more on metric information about the graph (the bond lengths).

In general, the combinatorial problem (19) is very hard and cannot be solved in a closed form. Nevertheless exact result for finite $t$ can always be obtained from (19) using a computer algebra system such as Maple [32]. To this end, one has to represent $\operatorname{tr} U^{t}$ as a multivariate polynomial of degree $t$ in the variables $\exp \left(\mathrm{i} k L_{i}\right)$, i.e.

$$
\operatorname{tr} U^{t}=\sum_{\mathcal{P}_{t}} c_{\mathcal{P}}\left(\exp \left(\mathrm{i} k L_{1}\right)\right)^{p_{1}}\left(\exp \left(\mathrm{i} k L_{2}\right)\right)^{p_{2}} \ldots,
$$

where $\mathcal{P}_{t}$ runs over all partitions of $t$ into non-negative integers $t=p_{1}+p_{2}+\ldots[9]$. 

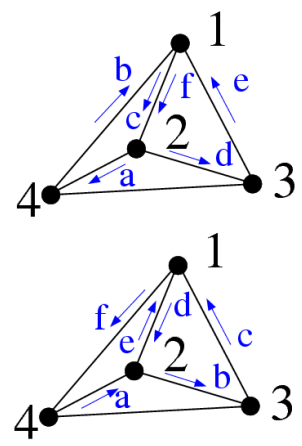
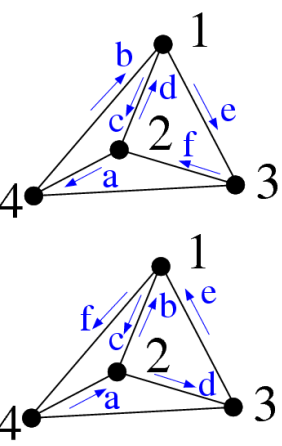
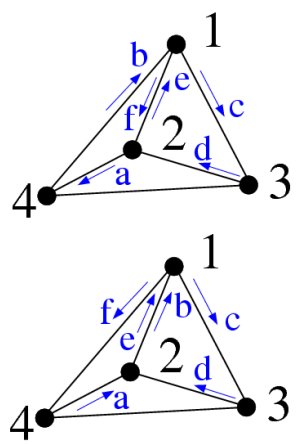

Fig. 3. The family $\mathcal{L}$ of isometric orbits $\mathcal{F}_{6}$ of period $t_{p}=6$ and length $L_{p}=2 l_{1,2}+$ $l_{1,4}+l_{1,3}+l_{2,3}+l_{2,4}$ for the tetrahedron. The various orbits (6 in total) are indicated with the sequence of letters associated with the arrows.

The form factor is then simply given as

$$
K(t / 2 B)=\sum_{\mathcal{P}_{t}}\left|c_{\mathcal{P}}\right|^{2}
$$

The task of finding the coefficients $c_{\mathcal{P}}$ can be expressed in Maple with standard functions. In Fig. 4 we compare the results of (21) with direct numerical averages for fully connected graphs with $V=4$ and $V=5$ vertices with and without magnetic field breaking the time-reversal symmetry. The results agree indeed to a high precision. Although this could be regarded merely as an additional confirmation of the numerical procedures used in [1], we see the main merit of (21) in being a very useful tool for trying to find the solution of (19) in a closed form.

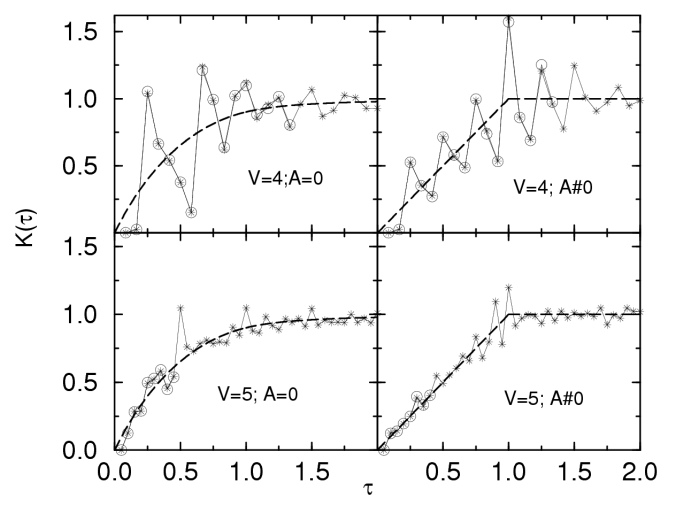

Fig. 4. Form factor of $U$ for regular graphs with $V=4$ vertices (top) and $V=5$ vertices (bottom). In the right parts an additional magnetic field destroyed time-reversal symmetry. Circles: exact results obtained from (19). Stars: numerical average over 2000 values of $k$. The solid line is to guide the eye. The prediction of the appropriate random matrix ensemble is shown with dashed lines. 


\section{Wave function statistics}

Following the quantization outlined in Sect. 2 a quantum wave function is defined as a set of $2 B$ complex amplitudes $a_{d}$, normalized according to $\sum_{d}\left|a_{d}\right|^{2}=1$. Here we will care about stationary solution satisfying Eq. (5) (i.e. eigenstates of the graph with corresponding wavelength $k$ ). The standard localization measure is the inverse participation ratio (IPR) which is defined as

$$
\mathcal{I}=\sum_{d=1}^{2 B}\left|a_{d}\right|^{4}
$$

Ergodic states which occupy each directed bond with the same probability have $\mathcal{I}=1 / 2 B$ and up to a constant factor depending on the presence of symmetries this is also the RMT prediction. In the other extreme $\mathcal{I}=0.5$ indicates a state which is restricted to a single bond only, i.e. the greatest possible degree of localization. Some representative eigenstates are shown in Fig. 5.

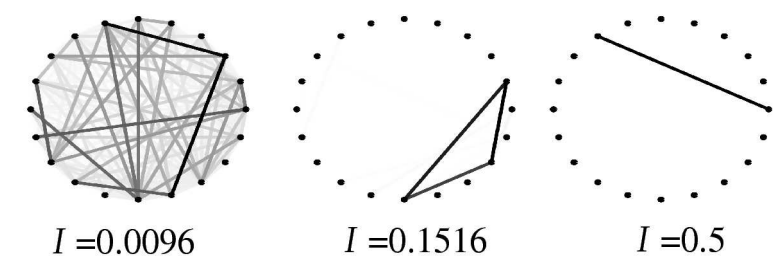

Fig. 5. Representative eigenstates for a fully connected graph with $V=10$. The corresponding IPR's are (from left to right) $\mathcal{I}=\frac{1}{104} \approx 0.0096 ; \mathcal{I}=\frac{1}{6} \approx 0.1516$; $\mathcal{I}=\frac{1}{2}=0.5$.

The key theoretical idea discussed and applied in several recent works [33-35] is that wave function intensities in a complex system can often be separated into a product of short-time and long-time parts, the latter being a random variable. On the other hand the short time part can be evaluated using information about classical dynamics. Specifically we have that the probability amplitude $A_{d}$ to return to the original state $|d\rangle$ is

$$
A_{d} \equiv\left\langle d\left|U^{t}\right| d\right\rangle=\sum_{m}|\langle d \mid m\rangle|^{2} \exp \left(-\mathrm{i} \epsilon_{m} t\right)
$$

The return probability is then

$$
P_{d}(t) \equiv\left|A_{d}\right|^{2}=\sum_{m, n}|\langle d \mid m\rangle|^{2}|\langle d \mid n\rangle|^{2} \exp \left(\mathrm{i}\left(\epsilon_{m}-\epsilon_{n}\right) t\right) .
$$

Averaging over initial states and over time (typically larger than the Heisenberg time $t_{\mathrm{H}}=2 \pi / \Delta=2 B$ ) we get

$\left\langle{\overline{P_{d}(t)}}^{t}\right\rangle_{d} \equiv\left\langle\frac{1}{2 B} \sum_{t=1}^{2 B} P_{d}(t)\right\rangle_{d}=\frac{1}{2 B} \sum_{t=1}^{2 B} P(t)=\left\langle\sum_{m}|\langle d \mid m\rangle|^{4}\right\rangle_{d} \equiv\langle\mathcal{I}\rangle_{d}$,

where $\bar{\ldots}^{t}$ indicates an average over time and $\langle\ldots\rangle_{d}$ over initial states. Above 
$P(t)$ indicates the averaged (over initial states) return probability. In the last equality we had used the fact that due to time-average the off-diagonal terms averaged out to zero. Equation (25) expresses the mean IPR in terms of the quantum return probability (RP), averaged over time and initial states. The next step is to argue that the quantum short-time dynamics, can be described by the classical time evolution (see Fig. 6). The latter can be approximated semiclassically quite well based only on period-two PO's which correspond to trajectories which bounce back and forth between two vertices. These type of orbits have the lowest

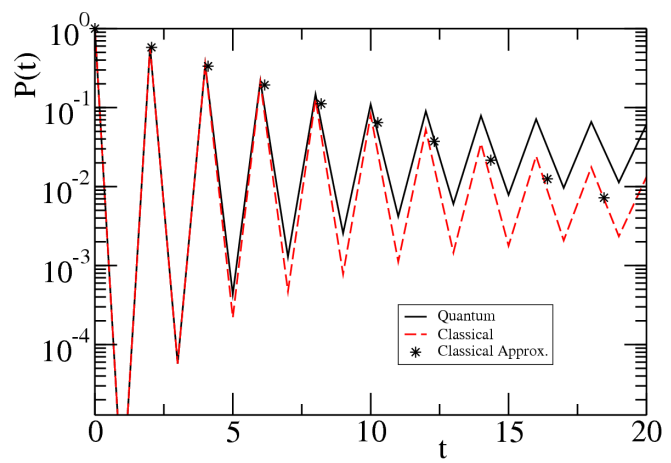

Fig. 6. The quantum survival probability (solid line), the classical survival probability (dashed line) and the classical short time approximation $(\star)$ based only in the period-two orbits as it is given by Eq. (26).

Lyapunov exponent and it is expected to have the largest influence on eigenfunction localization because classical trajectories can cycle in their vicinity for a relatively long time and increase the RP beyond the ergodic average. The resulting survival probability is

$$
P(t)= \begin{cases}0, & t \text { odd } \\ \left(-1+\frac{2}{v}\right)^{4}, & t \text { even. }\end{cases}
$$

Indeed the period 2 orbits totally dominate the classical and quantum RP at short times as can be seen in Fig. 6. Including the contribution of these orbits only, Kaplan obtained a mean IPR which is by a factor $\sim v$ larger than the RMT expectation, in agreement with numerics [35]. Moreover, following the same line of argumentation as in [34] we get that the bulk of the IPR distribution scales as [13]

$$
\tilde{\mathcal{P}}(\mathcal{I} /\langle\mathcal{I}\rangle)=\langle\mathcal{I}\rangle \mathcal{P}(\mathcal{I})
$$

indicating that the whole bulk of $\mathcal{P}(\mathcal{I})$ is effectively determined by the least unstable orbits. This result can be nicely verified from the numerical data presented in Fig. 7.

With all this evidence for their prominent role in wave function localization, one clearly expects to see strong scarring on the period-two orbits. Such states 


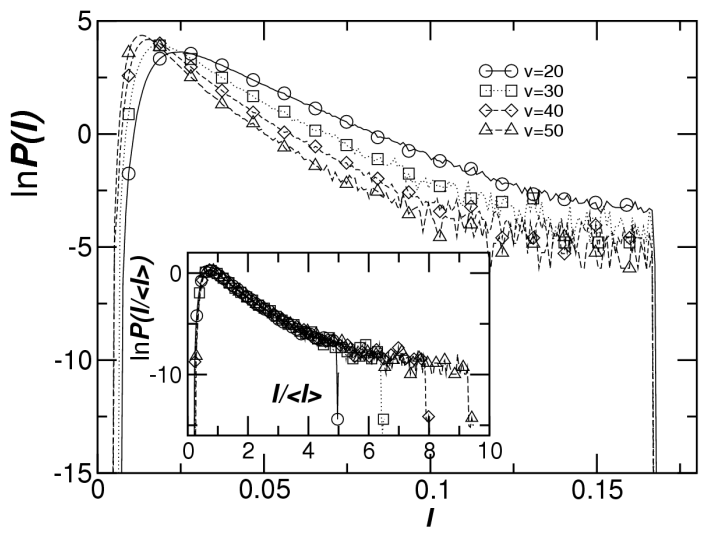

Fig. 7. Probability distribution $\mathcal{P}(\mathcal{I})$ of the inverse participation numbers, showing a step-like cutoff at $\mathcal{I}=1 / 6$ that can be attributed to scarring on triangular orbits. In the inset we report the rescaled distribution $\tilde{\mathcal{P}}(\mathcal{I} /\langle\mathcal{I}\rangle)$. A nice scaling is observed.

would essentially be concentrated on two directed bonds and give rise to $\mathcal{I} \sim 1 / 2$. However, in this region $\mathcal{P}(\mathcal{I})$ is negligible (see Fig. 7 ). We conclude therefore that the shortest and least unstable orbits of our system produce no visible scars. Let us note that the same applies also to the value $\mathcal{I}=1 / 4$ expected from the $\mathrm{V}$-shaped orbits of Fig. 2. In fact $\mathcal{P}(\mathcal{I})$ has an appreciable value only for $\mathcal{I} \leq 1 / 6$ (Fig. 7 ). The position of this cutoff precisely coincides with the IPR expected for states which are scarred by triangular orbits. They occupy six directed bonds since, due to time-reversal symmetry, scarring on a PO and its reversed must coincide. Indeed a closer inspection shows that the vast majority of states at $\mathcal{I} \approx 1 / 6$ look like the example shown in Fig. 5. Of course the step at $\mathcal{I}=1 / 6$, which is present for any graph size $V$, is incompatible with the scaling of $\mathcal{P}(\mathcal{I})$ mentioned above and indeed this relation breaks down in the tails at the expected points (the inset in Fig. 7).

These results [13] provide clear evidence for the fact that enhanced wave function localization due to the presence of short unstable orbits and strong scarring can in principle rely on completely unrelated mechanisms and can also leave distinct traces in statistical measures such as the distribution of inverse participation ratios. As a matter of fact in [13] we were able to identify a necessary and sufficient condition for the energies of perfect scars

$$
\left(k L_{d}\right) \bmod \pi=0 \quad \forall d \in p,
$$

where $d$ is a directed bond which belongs to the specific PO $p$. Equation (28) is reminiscent of a simple Bohr-Sommerfeld quantization condition $k L_{p}=2 n \pi$, as it applies, e.g., to strong scars in billiards. However, there is an important difference: not only Eq. (28) does require quantization of the total action $k L_{p}$ of the scarred orbit, it also implies action quantization on all the visited bonds $d$. This stronger condition can only be met if the lengths of all bonds on $p$ are rationally related. 
As in general the bond lengths are incommensurate there are no perfect scars for generic graphs. Nevertheless, for incommensurate bond lengths Eq. (28) can be approximated with any given precision and then visible scars are expected [13].

\section{Conclusions and outlook}

We have reviewed some of our results on the statistical properties of eigenvalues and eigenfunctions of the unitary quantum time evolution operator derived from quantum graphs. We have concentrated on fully connected quantum graphs. For this family of graphs, the gap $\Delta_{g}$ between the two maximum eigenvalues of the classical evolution operator approaches 1 as the number of directed bonds increases, thus satisfying the (sufficient) condition [16] for a graph in order to show universal spectral statistics.

At the same time, we show that the existing scar theory does not explain the appearance of visible scars (super-scars). As a matter of fact our numerical data indicated that enhanced wave function localization due to short unstable orbits and strong scarring are not the same thing.

Quantum graphs were proven throughout the years very useful models. They allowed us to gain a good understanding of the spectrum and eigenfunctions properties of quantum systems with underlying classical chaotic dynamics. Semiclassics on graphs is exact, and various quantum mechanical quantities can be written in terms of classical periodic orbits. These studies and their conclusions are by now well documented in the quantum chaos literature. But quantum chaology has various other challenges that wait to be addressed. Among them is a quantum mechanical theory of dynamical evolution which is still a missing chapter. Quantum dissipation, dephasing and irreversibility (also used in the framework of fidelity studies in quantum computation) of quantum chaotic motion are notions, which are related with specific aspects of this evolution. It is our belief that quantum graphs can play a prominent role in this ultimate challenge: to develop a general theory for the time evolution of quantum systems with underlying classical chaotic behavior.

\section{Acknowledgments}

We would like to express our gratitude to Prof. Uzy Smilansky to whom we owe our interest in the subject, and to Dr. Holger Schanz who contributed a great deal to the results discussed in this contribution.

\section{References}

[1] T. Kottos, U. Smilansky, Phys. Rev. Lett. 79, 4794 (1997); Ann. Phys. 274, 76 (1999).

[2] H. Schanz, U. Smilansky, Philos. Mag. B 80, 1999 (2000).

[3] G. Berkolaiko, J. Keating, J. Phys. A 32, 7827 (1999). 
[4] F. Barra, P. Gaspard, J. Stat. Phys. 101, 283 (2000); F. Barra, P. Gaspard, Phys. Rev. E 63, 066215 (2001).

[5] E. Akkermans, A. Comtet, J. Desbois, G. Montambaux, C. Texier, Ann. Phys. 284, 10 (2000)

[6] H. Schanz, U. Smilansky, Phys. Rev. Lett. 84, 1427 (2000).

[7] T. Kottos, U. Smilansky, Phys. Rev. Lett. 85, 968 (2000); J. Phys. A 85, 968 (2003).

[8] G. Tanner, J. Phys. A 33, 3567 (2001).

[9] T. Kottos, H. Schanz, Physica E 9, 523 (2001).

[10] L. Kaplan, Phys. Rev. E 64, 036225 (2001).

[11] G. Berkolaiko, H. Schanz, R.S. Whitney, Phys. Rev. Lett. 88, 104101 (2002).

[12] R. Blümel, Yu. Dabaghian, R.V. Jensen, Phys. Rev. Lett. 88, 044101 (2002); Yu. Dabaghian, R. Blümel, Phys. Rev. E 68, 055201 (2003).

[13] H. Schanz, T. Kottos, Phys. Rev. Lett. 90, 234101 (2003); G. Berkolaiko, J.P. Keating, B. Winn, Phys. Rev. Lett. 91, 134103 (2003).

[14] H. Schanz, M. Puhlmann, T. Geisel, Phys. Rev. Lett. 91, 134101 (2003).

[15] S. Gnutzmann, U. Smilansky, J. Weber, J. Phys. A 14, S61 (2004).

[16] S. Gnutzmann, A. Altland, Phys. Rev. Lett. 93, 194101 (2004).

[17] O. Hul, S. Bauch, P. Pakoński, N. Savytskyy, K. Życzkowski, L. Sirko, Phys. Rev. E 69, 056205 (2004).

[18] Special Issue on Quantum Graphs, Ed. P. Kuchment, Waves in Random Media 14, (2004).

[19] M. Puhlmann, H. Schanz, T. Kottos, T. Geisel, Europhys. Lett. 69, 313 (2005).

[20] O. Bohigas, M.J. Giannoni, C. Schmit, Phys. Rev. Lett. 52, 1 (1984).

[21] M.V. Berry, Proc. R. Soc. London A 400, 229 (1985).

[22] N. Argaman, F.-M. Dittes, E. Doron, J.P. Keating, A.Yu. Kitaev, M. Sieber, U. Smilansky, Phys. Rev. Lett. 71, 4326 (1993).

[23] D. Cohen, H. Primack, U. Smilansky, Ann. Phys. 264, 108 (1998).

[24] J.P. Roth, in: Lectures Notes in Mathematics, Eds. A. Dold, B. Eckmann, Vol. 1096, Springer-Verlag, Berlin 1984, p. 521.

[25] M.L. Mehta, Random Matrices and the Statistical Theory of Energy Levels, Academic Press, New York 1990.

[26] S. Sridhar, Phys. Rev. Lett. 67, 785 (1991).

[27] P.B. Wilkinson, T.M. Fromhold, L. Eaves, F.W. Sheard, N. Miura, T. Takamasu, Nature 380, 608 (1996).

[28] A. Kudrolli, M.C. Abraham, J.P. Gollub, Phys. Rev. E 63, 026208 (2001).

[29] J.P. Bird, R. Akis, D.K. Ferry, Phys. Scr. T 90, 50 (2001).

[30] C. Gmachl, E.E. Narimanov, F. Capasso, J.N. Baillargeon, A.Y. Cho, Opt. Lett. 27, 824 (2002).

[31] U. Smilansky, in: Proc. 1989 Les Houches Summer School on Chaos and Quantum Physics, Eds. M.J. Giannoni, A. Voros, J. Zinn-Justin, North-Holland, Amsterdam 1991, p. 371. 
[32] M. Kofler, Maple: An Introduction and Reference, Addison-Wesley, Harlow 1997.

[33] E.J. Heller, Phys. Rev. Lett. 53, 1515 (1984).

[34] L. Kaplan, E.J. Heller, Ann. Phys. 264, 171 (1998).

[35] L. Kaplan, Phys. Rev. E 64, 036225 (2001). 